01

\title{
Конечно-элементное моделирование одноосного растяжения образцов из функционально-градиентного материала с использованием мультилинейной модели пластичности
}

\author{
(C) В.Ш. Суфияров, ${ }^{1}$ А.В. Орлов, ${ }^{1}$ Е.В. Борисов, ${ }^{1}$ И.А. Полозов, ${ }^{1}$ А.А. Попович, ${ }^{1}$ М.О. Чуковенкова, ${ }^{2,}$ \\ А.В. Соклаков, Д.С. Михалюк ${ }^{2}$ \\ ${ }^{1}$ Санкт Петербургский политехнический университет Петра Великого, \\ 195251 Санкт-Петербург, Россия \\ ${ }^{2}$ АО „Центр инженерно-физических расчетов и анализа“, \\ 195197 Санкт-Петербург, Россия \\ 『e-mail: maria.chukovenkova@multiphysics.ru
}

Поступило в Редакцию 24 января 2019 г. В окончательной редакции 16 июля 2020 г. Принято к публикации 17 июля 2020 г.

\begin{abstract}
Предложен подход к численному моделированию механических свойств функционально-градиентных материалов методом конечных элементов с использованием мультилинейной модели пластичности. Используя данный подход, проведено моделирование экспериментов по растяжению однородных образцов, изготовленных при двух различных мощностях лазера, а также составных образцов на базе рассмотренных материалов. Показана корреляция между данными, полученными методом численного моделирования, и экспериментальными данными. Исследовано влияние формы и размера вставок в составных образцах на механические свойства, и определено, что, изменяя размер и форму вставок, можно менять механические характеристики образца. Установлено, что мультилинейная модель пластичности дает наилучшее согласование с экспериментальными данными, и в отличие от билинейной модели пластичности является более информативной.
\end{abstract}

Ключевые слова: аддитивные технологии, функционально-градиентный материал, метод конечных элементов, Inconel 718.

DOI: 10.21883/JTF.2021.01.50268.21-19

\section{Введение}

Аддитивное производство имеет множество преимуществ по сравнению с традиционными методами производства. Одним из преимуществ является возможность создания металлических конструкций со сложной геометрией, включая, например, ячеистые и топологическиоптимизированные структуры, внутренние каналы охлаждения, которые невозможно получить с помощью механической обработкой или литьем [1]. В настоящее время с помощью аддитивных методов можно изготавливать детали из широкого спектра материалов, например, из сплавов на основе никеля, титана, алюминия, стали и др. [2-4]. Новейшим трендом в аддитивном производстве является производство функционально-градиентных материалов (ФГМ) с различной микроструктурой [5], различным химическим составом [6], включая объекты, состоящие из нескольких материалов, а также имеющих градиентную пористость, которую получают, используя оптимизированные ячеистые структуры [7]. Аддитивное производство ФГМ с учетом микроструктуры предлагает возможность для производства конструкций микроструктуру, например, с различным размером зерна, кристаллографической ориентацией, специально учитывающие условия нагружения конструкции $[8,9]$.
Расчет конструкций из функционально-градиентных материалов методом конечных элементов (МКЭ) предполагает различные подходы в решении, позволяющие моделировать неоднородность свойств градиентного материала. В работах $[10,11]$ предложен способ, основанный на присвоении различных свойств материала в последовательных слоях конечно-элементной сетки. Подобный подход к расчетам является затратным по вычислительным ресурсам. В работе [12] рассмотрено моделирование механических характеристик ФГМ на основании экспериментальных данных с использованием билинейной модели пластичности путем введения параметра, характеризующего неоднородность свойств материала. Этот способ позволяет приблизить результаты численного моделирования к результатам эксперимента.

Цель настоящей работы - численное моделирование механических свойств образцов из ФГМ на основании имеющихся экспериментальных данных с использованием мультилинейной модели пластичности с целью установления корреляции между расчетными и экспериментальными данными прочностных характеристик и дальнейшей валидации полученной численной модели. В рамках настоящей работы поставлены следующие задачи: моделирование эксперимента для однородных материалов; моделирование составных образцов на базе 
имеющихся однородных материалов; исследование влияния формы и размера вставок в составных образцах; сравнение результатов, полученных с использованием мультилинейной модели пластичности, с результатами, полученными для билинейной модели пластичности.

\section{1. Расчетная модель}

Для моделирования использовался пакет конечноэлементного анализа ANSYS 19.1. Подготовка геометрической модели произведена в CAD-модуле ANSYS SpaceClaim. Построение конечно-элементной сетки выполнено при помощи ANSYS Meshing. Постановка задачи, выполнение расчетов и обработка полученных результатов проведены в модуле ANSYS Mechanical.

Модель пластичности, использованная для моделирования одноосного растяжения, - мультилинейная. Для численного моделирования использовались механические характеристики, полученные из испытаний на растяжение образцов из однородных материалов. Для мультилинейной модели пластичности требуемыми данными являются: модуль Юнга $E$, коэффициент Пуассона $\mu$ и зависимость пластического напряжения от относительной пластической деформации $\varepsilon$.

Для описания пластического поведения материала требуется зависимость истинных напряжений от деформаций. Напряжения, полученные из эксперимента, являются инженерными и не учитывают изменения поперечного сечения образца. Для пересчета инженерных напряжений в истинные использовались формулы (1) и (2):

$$
\begin{gathered}
\sigma_{t}=\sigma_{b} \cdot\left(1+\varepsilon_{b}\right), \\
\varepsilon_{t}=\ln \left(1+\varepsilon_{b}\right),
\end{gathered}
$$

В настоящей работе использованы экспериментальные данные по растяжению образцов, изготовленных при
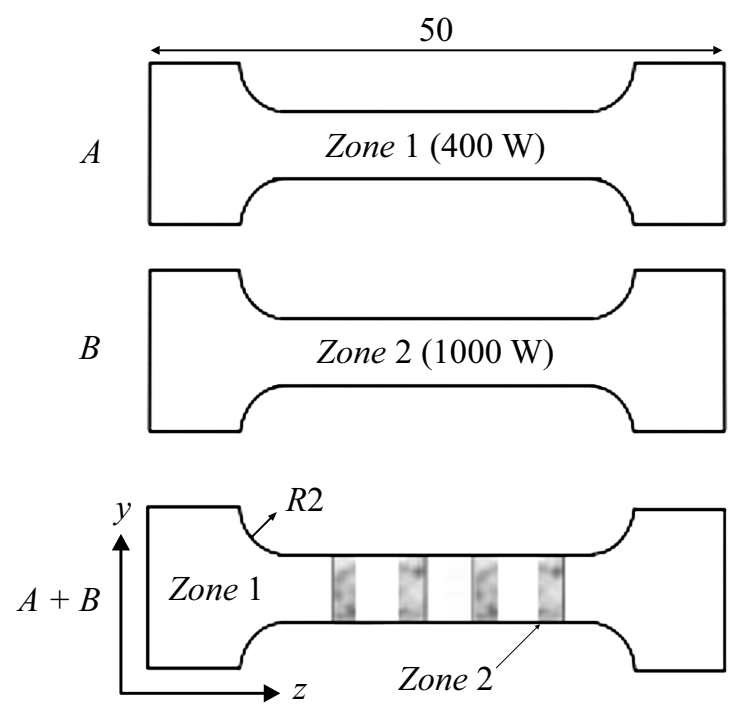

Рис. 1. Образцы из материалов $A, B$ и составной образец $A+B$.

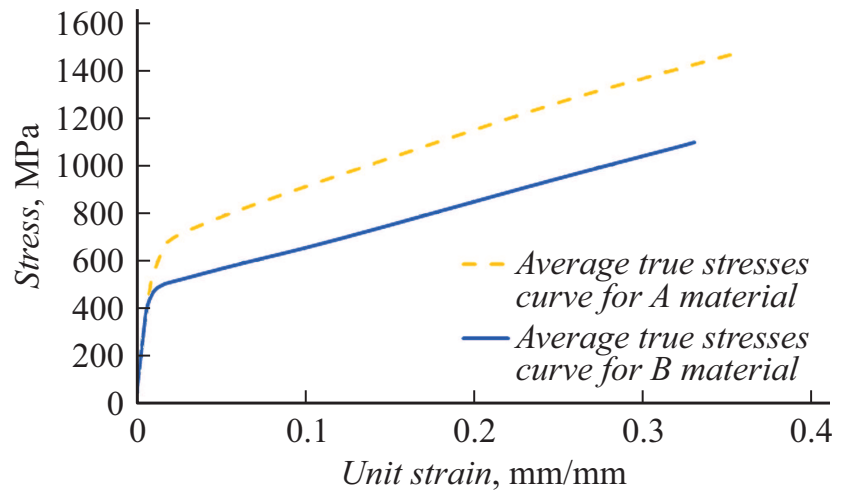

Рис. 2. Осредненные кривые истинных напряжений для материалов $A$ и $B$.

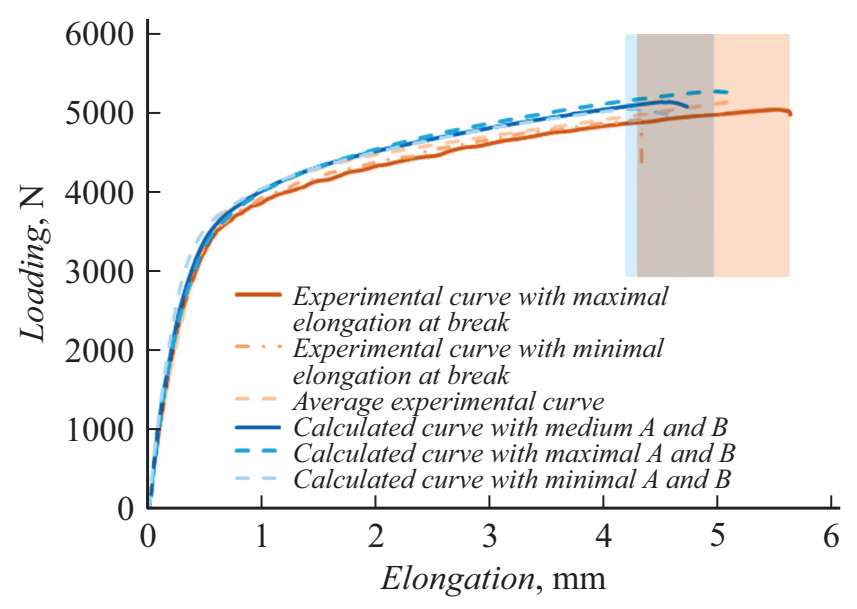

Рис. 3. Коридоры минимальных и максимальных расчетных и экспериментальных значений по растяжению составного образца (матрица A, 4 вставки - материал $B$ ).

двух различных мощностях лазера: $400 \mathrm{~W}$ (6 образцов) и $1000 \mathrm{~W}$ (6 образцов), далее по тексту материалы $A$ и $B$ соответственно. Также рассмотрены экспериментальные данные по растяжению составного образца, состоящего из двух материалов: основная матрица - материал, изготовленный при мощности лазера $400 \mathrm{~W}$ и четыре вставки из материала, изготовленного при мощности лазера $1000 \mathrm{~W}$ (5 образцов), далее - составной образец из материалов $A$ и $B$ (рис. 1).

Пересчет в истинные напряжения с последующим получением мультилинейной кривой был проведен для всех экспериментальных кривых для материалов $A$ и $B$, а также получены осредненные кривые истинных напряжений (рис. 2).

Полученные экспериментально кривые деформирования для образцов из одинаковых материалов отличаются друг от друга по величине остаточного удлинения. Для моделирования поведения составных образцов использованы осредненные мультилинейные кривые, а также для определения коридора расчетных значений рассмотрены различные комбинации мультилинейных кривых: $A$ и $B$ 


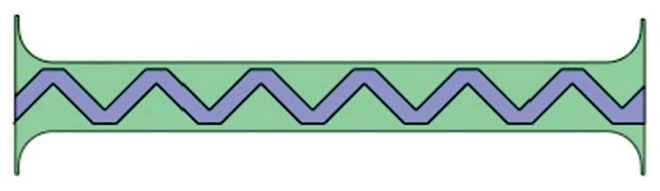

Insert 1

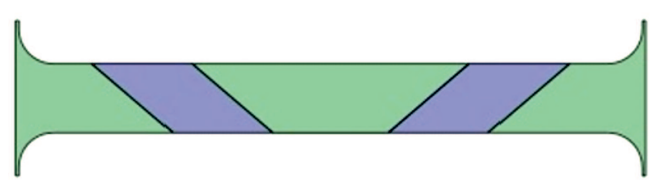

Insert 2

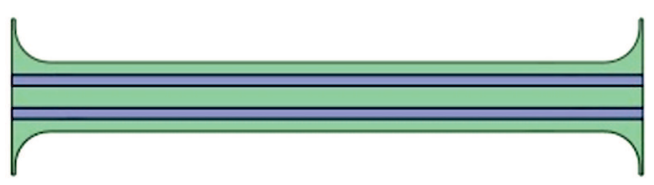

Insert 3

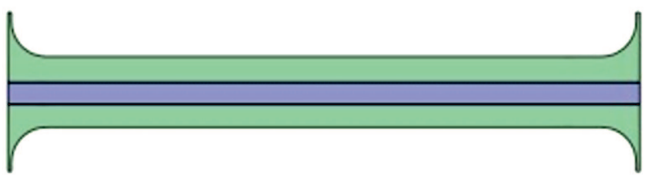

Insert 4

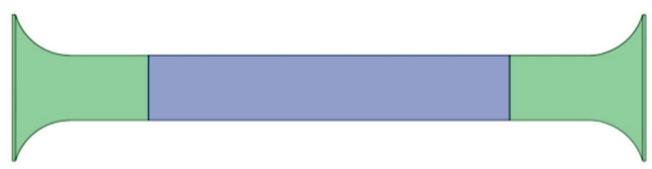

Insert 5

Рис. 4. Геометрия составных образцов при содержании материала $A 30 \%$.

с максимальным удлинением при разрыве, $A$ и $B$ с минимальным удлинением при разрыве.

Переходная зона между участками с крупнозернистой структурой, напечатанными при высокой мощности, и мелкозернистой, напечатанной на низкой мощности, имеет размер $\sim 500 \mu \mathrm{m}[13]$, что позволяет авторам сделать допушение о четкой границе раздела материалов в рамках рассмотренных конечно-элементных моделей.
На рис. 3 показаны коридоры расчетных значений (синяя область (в online-версии)): минимальное значение удлинения при разрыве составляет $4.15 \mathrm{~mm}$ (образец с минимальными удлинениями $A$ и $B$ ), максимальное $4.95 \mathrm{~mm}$ (образец с максимальными удлинениями $A$ и $B$ ). Удлинение при разрыве образца с осредненными свойствами материалов составляет $4.55 \mathrm{~mm}$.

Коридор экспериментальных значений выделен красным цветом (в online-версии), минимальное значение удлинения при разрыве составляет $4.3 \mathrm{~mm}$, максимальное - $5.5 \mathrm{~mm}$. Среднее экспериментальное удлинение при разрыве составляет $5.15 \mathrm{~mm}$. Совпадение результатов расчетов с результатами экспериментов соответствует области пересечения коридоров.

Сравнение результатов моделирования с экспериментальными данными проведено для следующего набора кривых: экспериментальная кривая с максимальным удлинением при разрыве (далее - экспериментальная кривая максимальная) и расчетная кривая с $A$ и $B$, имеющими максимальное удлинение при разрыве (далее - $A$ и $B$ максимальные); экспериментальная кривая с минимальным удлинением при разрыве (далее экспериментальная кривая минимальная) и расчетная кривая с $A$ и $B$, имеющими минимальное удлинение при разрыве (далее - $A$ и $B$ минимальные); осредненная экспериментальная кривая и расчетная кривая с осредненными значениями для $A$ и $B$ представлены в таблице.

Используя осредненные экспериментальные кривые для материалов, мы провели моделирование образцов со вставками пяти различных форм, показанных на рис. 4. Объемное содержание материала вставки в матрице варьируется от 10 до 50\%. Матрица состоит из материала $B$, вставки - из материала $A$. Исследовано влияние формы и размера вставки на прочностные характеристики образца. Экспериментальные исследования влияния формы и размера вставки не проводились. $a$

Equivalent stress
unit: $\mathrm{MPa}$
\begin{tabular}{|l}
1352.7 max \\
1300 \\
1100 \\
1100 \\
1000 \\
900 \\
700 \\
500 \\
300 \\
100 \\
10 \\
2.3747 min
\end{tabular}

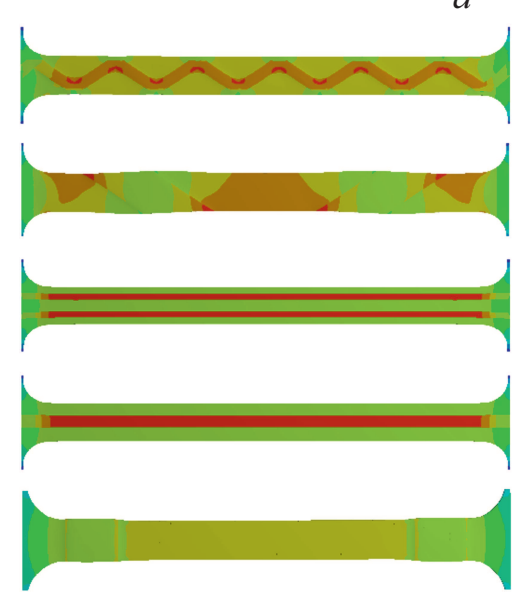

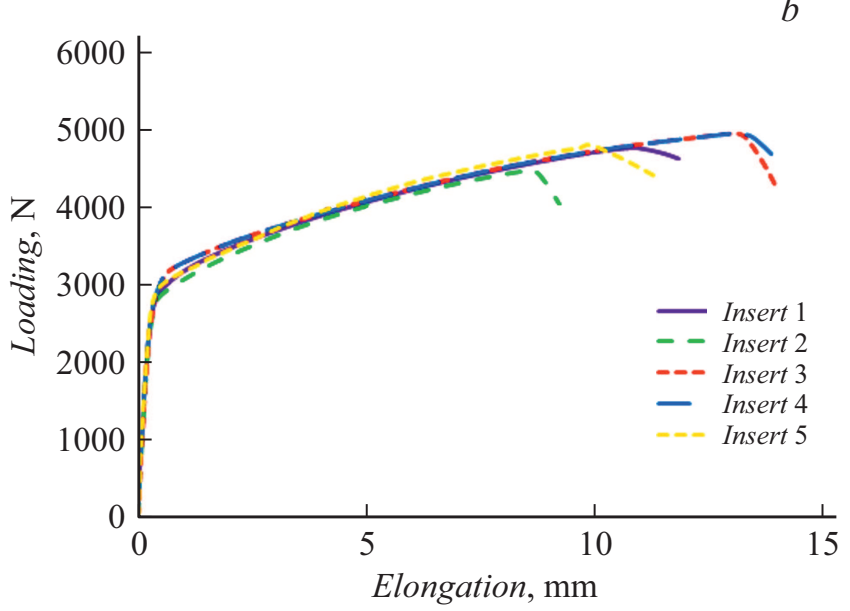

Рис. 5. $a$ - поля эквивалентных напряжений при растяжении образцов со вставками при удлинении на $8 \mathrm{~mm} ; b-$ диаграммы растяжения образцов со вставками типов $1-5$. 
Сравнение расчетных данных с экспериментальными для составных образцов

\begin{tabular}{|c|c|c|c|c|}
\hline \multicolumn{5}{|c|}{ Матрица A, 4 вставки - материал $B$} \\
\hline \multirow[b]{2}{*}{ Кривые } & \multicolumn{4}{|c|}{ Отклонение, \% } \\
\hline & $\begin{array}{l}\text { по модулю } \\
\text { Юнга, } \\
E\end{array}$ & $\begin{array}{c}\text { по пределу } \\
\text { текучести, } \\
\sigma_{0,2}\end{array}$ & $\begin{array}{c}\text { по пределу } \\
\text { прочности, } \\
\sigma_{B}\end{array}$ & $\begin{array}{c}\text { по остаточному } \\
\text { удлинению, } \\
\delta\end{array}$ \\
\hline $\begin{array}{l}\text { Экспериментальная кривая, } \\
\text { максимальная и расчетная кривые } \\
\text { с } A \text { и } B \text { максимальными }\end{array}$ & 6.1 & 4.2 & 4.5 & 10.0 \\
\hline $\begin{array}{l}\text { Экспериментальная кривая, } \\
\text { минимальная и расчетная кривые } \\
\text { с } A \text { и } B \text { минимальными }\end{array}$ & 10.7 & 12.8 & 2.3 & 3.5 \\
\hline $\begin{array}{l}\text { Осредненная экспериментальная и } \\
\text { расчетная кривые с осредненными } \\
A \text { и } B\end{array}$ & 2.9 & 3.7 & 0.2 & 11.7 \\
\hline $\begin{array}{l}\text { Осредненная экспериментальная } \\
\text { кривая и расчетная кривая, } \\
\text { полученная с использованием } \\
\text { билинейной модели } \\
\text { пластичности [12] }\end{array}$ & 6.0 & 4.0 & 5.0 & $\begin{array}{c}\text { билинейная } \\
\text { модель } \\
\text { пластичности } \\
\text { не дает } \\
\text { информации } \\
\text { об остаточном } \\
\text { удлинении }\end{array}$ \\
\hline
\end{tabular}
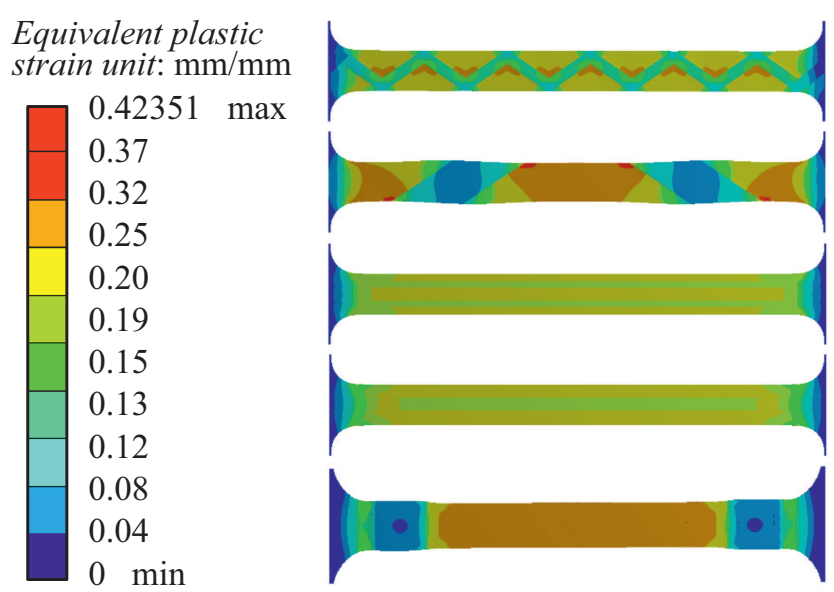

Рис. 6. Поля эквивалентных пластических деформаций при растяжении образцов со вставками при удлинении на $8 \mathrm{~mm}$.

На рис. 5, $а$ показано распределение эквивалентных напряжений для образцов со вставками при удлинении на $8 \mathrm{~mm}$. Объемное содержание материала вставок в образце - 30\%. В образцах со вставками типа 1 и 2 возникают концентраторы напряжений, вызванные геометрической формой вставок. Остаточное удлинение образцов с такими вставками меньше, чем у образцов со вставками типа 3 и 4 (рис. 5, b). Поля эквивалентных пластических и эквивалентных полных деформаций для образцов со вставками показаны на рис. 6 и 7.
Equivalent total strain unit: $\mathrm{mm} / \mathrm{mm}$

$0.43722 \max$
0.350
0.310
0.218
0.215
0.180
0.150
0.050
$2.9635 \cdot 10^{-5} \mathrm{~min}$

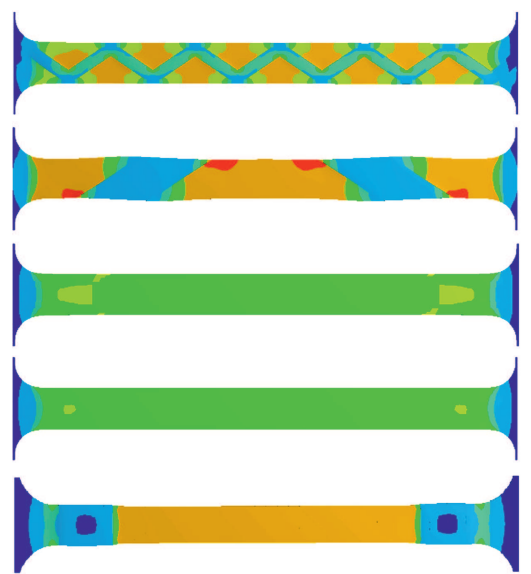

Рис. 7. Поля эквивалентных полных деформаций при растяжении образцов со вставками при удлинении на $8 \mathrm{~mm}$.

Для определения влияния объемного содержания материала вставки на механические свойства рассмотрены вставки типов 2 и $4-$ с минимальным и максимальным пределами прочности (рис. 8, 9).

Объемная доля материала вставки для типа 2 оказывает влияние на величину остаточного удлинения. Увеличение объемной доли материала вставки приводит к уменьшению величины остаточного удлинения. Предел текучести и предел прочности не зависят от объемной доли материала вставки. 

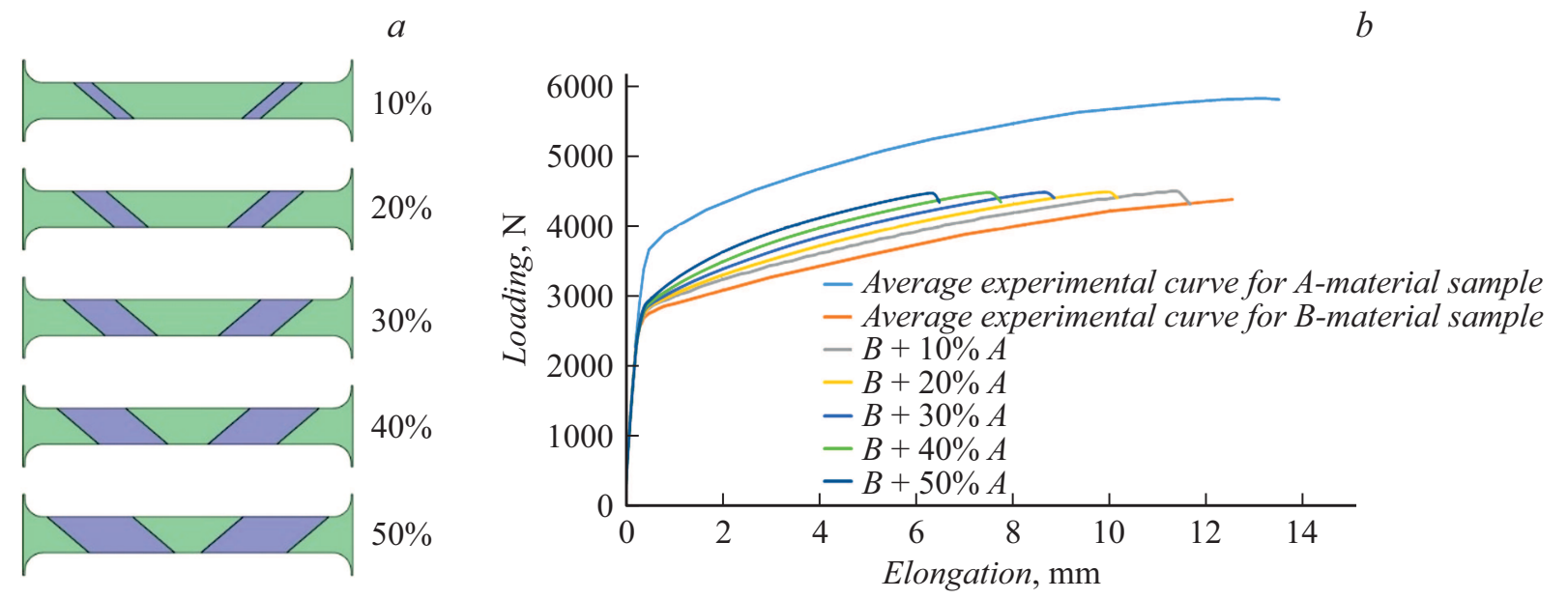

Рис. 8. $a$ - вставка 2 при различном процентном содержании материала $A$ в матрице $\mathrm{B} ; b-$ расчетные кривые деформирования для этих значений содержания.
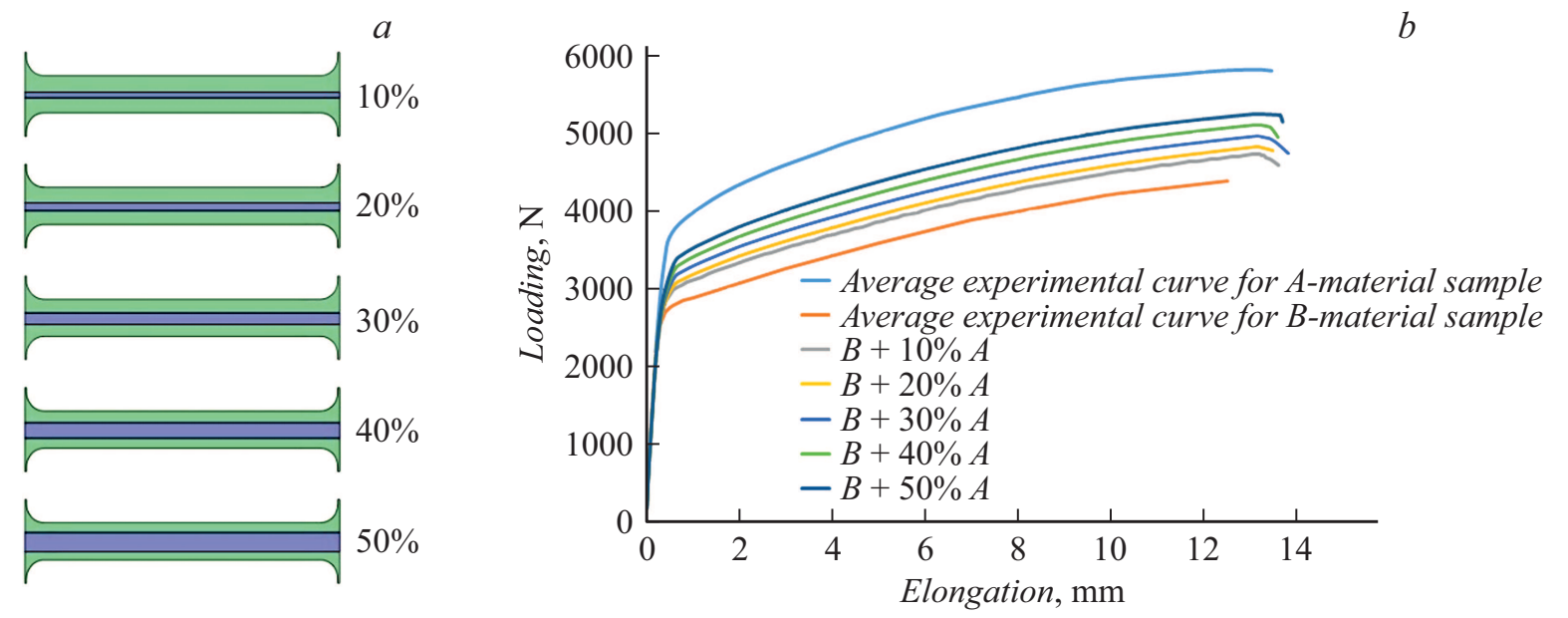

Рис. 9. $a-$ вставка 4 при различном процентном содержании в матрице; $b-$ расчетные кривые деформирования для этих значений содержания.

При увеличении объемной доли материала вставки типа 4 наблюдается увеличение предела прочности и предела текучести при неизменном остаточном удлинении.

\section{Заключение}

В работе проведено численное моделирование эксперимента по растяжению образцов для однородных материалов и составных образцов на базе имеющихся однородных материалов с использованием мультилинейной модели пластичности.

В результате сравнения результатов, полученных путем моделирования однородного образца с экспериментальной кривой, выявлено отклонение $0.05 \%$ по пределу текучести, $0.07 \%$ по пределу прочности и $3.57 \%$ по остаточному удлинению. Следовательно, использование мультилинейной модели пластичности позволяет с высокой точностью описать характер пластичности, возникающей в материале.

Сравнение результатов моделирования составного образца с экспериментальными данными показало отклонение $2.9 \%$ по модулю упругости, $3.7 \%$ по пределу текучести, $0.2 \%$ по пределу прочности и $11.7 \%$ по остаточному удлинению. Отклонение при использовании билинейной модели пластичности составило 6\% по модулю упругости, 4\% по пределу текучести, 5\% по пределу прочности, данных об остаточном удлинении билинейная модель пластичности не дает, что говорит о предпочтительности применения мультилинейной модели пластичности, которая позволяет получать более точные результаты при численном моделировании и является более информативной.

Проведено исследование по определению влияния формы и размера вставки в составном образце. Увеличение объемной доли материала вставок из более прочного материала приводит к увеличению прочностных харак- 
теристик образца. Наличие концентраторов напряжений, вызванных геометрическими формами вставок, приводит к уменьшению остаточного удлинения. Меняя форму и объемное содержание вставки, можно изменять механические свойства конструкции. Меняя форму и объемное содержание вставки, можно менять механические свойства конструкции.

\section{Финансирование работы}

Работа выполнена в рамках реализации федеральной целевой программы „Исследования и разработки по приоритетным направлениям развития научно-технологического комплекса России на 2014-2020 годы“ уникальный идентификатор проекта RFMEFI57817X0245.

\section{Конфликт интересов}

Авторы заявляют, что у них нет конфликта интересов.

\section{Список литературы}

[1] W.E. Frazier. J. Mater. Eng. Performance, 23 (6), 1917 (2014).

[2] A. Popovich, V. Sufiiarov, I. Polozov, E. Borisov, D. Masaylo, A. Orlov. Mater. Lett., 179, 38 (2016). DOI: 10.1016/j.matlet.2016.05.064

[3] A. Popovich, V. Sufiiarov, I. Polozov, E. Borisov, D. Masaylo, A. Orlov. Intern. J. Bioprinting, 2 (2), 187 (2016).

[4] A.A. Antonysamy, J. Meyer, P.B. Prangnell. Mater. Characterization, 84, 153 (2013).

[5] G.H. Loh, E. Pei, D. Harrison, M.D. Monzon. Additive Manufacturing, 23, 34 (2018). DOI: $10.1016 /$ j.addma.2018.06.023

[6] F. Hengsbach, P. Koppa, M.J. Holzweissig, M.E. Aydinoz, A. Taube, K.P. Hoyer, O. Starykov, B. Tonn, T. Niendorf, T. Troster, M. Schaper. Progress in Additive Manufacturing, 3, 1 (2018).

[7] J. Parthasarathy, B. Starly, S. Raman. J. Manufactur. Processes, 13 (2), 160 (2011). DOI: 10.1016/j.jmapro.2011.01.004

[8] V. Sufiyarov, E. Borisov, I. Polozov. Tsvetnye Metally, 7, 68 (2018). DOI: 10.17580/tsm.2018.07.11

[9] A.A. Popovich, V.Sh. Sufiiarov, E.V. Borisov, I.A. Polozov, D.V. Masaylo. Mater. Phys. Mechan., 38(1), 1 (2018). DOI: 10.18720/MPM.3812018_1

[10] G. Anlas, M.H. Santare, J. Lambros. Intern. J. Fracture, 104 (2), 131 (2000). DOI: 10.1023/A:1007652711735

[11] T. Fujimoto, N. Noda. J. American Ceramic Society, 84 (7), 1480 (2001). DOI: 10.1111/j.1151-2916.2001.tb00864.x

[12] A.A. Popovich, V.Sh. Sufiiarov, E.V. Borisov, A.V. Orlov, I.A. Polozov, D.V. Masaylo, M.O. Chukovenkova, A.V. Soklakov, D.S. Mikhaluk. Lett. Mater., 9(1), 97 (2019). DOI: 10.22226/2410-3535-2019-1-97-102

[13] V.A. Popovich, E.V. Borisov, A.A. Popovich, V.Sh. Sufiiarov, D.V. Masaylo, L. Alzina. Mater. Design, 114, 441 (2017). DOI: 10.1016/j.matdes.2016.10.075 